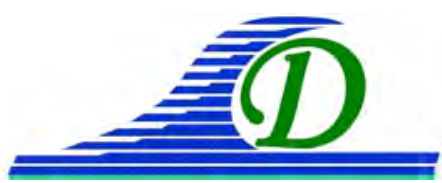
XIII ${ }^{\text {èmes }}$ Journées Nationales Génie Côtier - Génie Civil Dunkerque, 2-4 juillet 2014

DOI:10.5150/jngcgc.2014.049 (c) Editions Paralia CFL

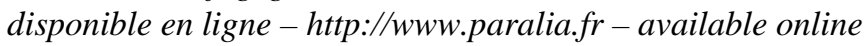

\title{
Modélisation numérique de l'hydrodynamique sédimentaire dans la baie d'Aigues-Mortes (Languedoc-Roussillon, France). Application à la géomorphodynamique et à la remise en suspension des sédiments rechargés sur les plages
}

\author{
Yann LEREDDE ${ }^{1}$, Héloöse MICHAUD ${ }^{1,2,3}$, Eric BERTHEBAUD ${ }^{1}$, Christine \\ LAUER-LEREDDE ${ }^{1}$, Patrick MARSALEIX ${ }^{2}$, Claude ESTOURNEL ${ }^{2}$, Bénédicte \\ GUERINEL $^{4}$, Sébastien THORIN ${ }^{5}$, Thibault SCHVARTZ ${ }^{5}$, Cécile RICHARD ${ }^{5}$
}

1. Géosciences Montpellier, Université Montpellier 2, CNRS, place Eugène Bataillon, 34095 Montpellier cedex 5, France.yann.leredde@um2.fr

2. Laboratoire d'Aérologie, Université de Toulouse, CNRS, 14, Avenue Edouard Belin, 31400 Toulouse, France. marp@aero.obs-mip.fr

3. SHOM, 14, Avenue Edouard Belin, 31400 Toulouse, France.

4. DREAL Languedoc-Roussillon, 58, avenue Marie de Montpellier, 34965

Montpellier, France. Benedicte.Guerinel@developpement-durable.gouv.fr

5. Créocéan, Agence de Montpellier. 128, avenue de Fès, 34080 Montpellier, France.

thorin@creocean.fr

\section{Résumé :}

Dans la baie d'Aigues-Mortes, les enjeux socio-économiques et patrimoniaux conduisent les communes du littoral et les gestionnaires à maintenir au mieux les usages en luttant contre les aléas auxquels ils sont soumis, notamment les aléas de contamination, d'érosion et de submersion. Pour lutter contre l'érosion, un rechargement massif de sable (un million de $\mathrm{m}^{3}$ sur un linéaire de côte de $10 \mathrm{~km}$ ) a été réalisé au début de l'année 2008. Le projet REPBAM (REchargements et Panaches sédimentaires dans la baie d'Aigues-Mortes) a pour objectif de qualifier et de quantifier par la mesure in situ et la modélisation numérique (i) l'évolution géomorphologique du littoral soumis à ce rechargement et (ii) l'impact de la remise en suspension des sédiments rechargés sur les écosystèmes côtiers, en particulier celui constitué par les herbiers de Posidonies.

Dans cette communication, nous nous focalisons sur les aspects de modélisation numérique de l'hydrodynamique sédimentaire allant de l'échelle de la plage du Petit Travers à l'échelle du plateau interne de la baie d'Aigues-Mortes. Cette modélisation, basée sur les travaux de MICHAUD et al. (2012), prend en compte les forçages des flux atmosphériques et des vagues sur les courants. Elle est tridimensionnelle et multiéchelles allant du plateau continental à la plage. Nous présentons ici des résultats pour la tempête de décembre 2008.

Mots-clés : Rechargement de plage, Panaches sédimentaires, Tempête, Turbidité, Herbiers de Posidonies, Modélisation numérique. 


\section{Thème 2 - Dynamique sédimentaire}

\section{Introduction}

Dans la baie d'Aigues-Mortes (voir figure 1), les enjeux socio-économiques sont énormes. La population d'estivants peut atteindre 300000 habitants. Le tourisme reste la ressource principale de la région. Les enjeux environnementaux sont également très importants. Les étangs Palavasiens et l'avant-côte avec la présence de fragiles herbiers de Posidonies, sont classés Natura 2000. Les petits métiers notamment de pêcheurs de tellines sont également bien présents dans la zone.

La préoccupation des communes du littoral et des gestionnaires est de maintenir au mieux tous ces usages en luttant contre les aléas auxquels ils sont soumis, notamment les aléas de contamination, d'érosion et de submersion. Pour lutter contre l'érosion, un rechargement massif de sable a ainsi été réalisé au début de l’année 2008. Le volume de sable injecté au sein de la cellule sédimentaire était de plus de un million de $\mathrm{m}^{3}$ sur un linéaire de côte de $10 \mathrm{~km}$.

Un rechargement dans de telles proportions est une nouveauté en France. L'évolution à moyen terme (sur 10 ans) et les effets indirects de ces rechargements, notamment sur les écosystèmes côtiers, sont ainsi en question. D’un point de vue morphodynamique, les zones de rechargement ont déjà largement évoluées sous l'effet principal des tempêtes. Depuis 2008, le suivi bathymétrique et granulométrique de la zone rechargée est assuré par la DREAL. D’un point de vue environnemental, ces tempêtes produisent également des panaches turbides dont les effets sur les herbiers de Posidonies sont au centre des préoccupations.

En effet, les herbiers de posidonies sont des écosystèmes très productifs et possèdent des fonctions écologiques et économiques très importantes dans la zone côtière méditerranéenne. La principale cause de dégradation des herbiers de par le monde est une réduction de la clarté de l'eau (SHORT \& WYLLIE-ECHEVERRIA, 1996). Cette réduction de l'intensité lumineuse peut provenir directement de l'augmentation de la turbidité de l'eau ou indirectement de la sédimentation sur les feuilles qui en résulte.

Afin de répondre aux préoccupations concrètes des gestionnaires du littoral quant au devenir et à l'impact de rechargements massifs de sédiments sur les plages en période de tempêtes, le projet REPBAM (REchargements et Panaches sédimentaires dans la Baie d'Aigues-Mortes), a proposé une approche méthodologique multidisciplinaire combinant

- des simulations numériques de l'échelle de la plage à celle du plateau interne,

- des mesures physiques en continu,

- des analyses des données bathymétriques et granulométriques,

- des observations et mesures biologiques ponctuelles permettant de mieux définir l'impact des panaches turbides sur les herbiers de Posidonies.

Dans cet article, nous montrons des premiers résultats de simulations numériques conduites pour la période de décembre 2008. 


\section{XIII ${ }^{\text {èmes }}$ Journées Nationales Génie Côtier - Génie Civil \\ Dunkerque, 2-4 juillet 2014}

Deux échelles sont présentes. La première est celle de la plage rechargée en elle-même dont la morphologie évolue au cours du temps et au grès des tempêtes. La seconde est celle du plateau interne soumis à l'influence des panaches turbides susceptibles de se former lors de la mise en suspension des sédiments rechargés, notamment pendant les tempêtes. Ces sédiments rechargés sont faiblement compactés et ne sont pas répartis de manière naturelle mais anthropique. Ils sont donc plus facilement remobilisables, ce qui confère à un tel site un intérêt remarquable pour la modélisation.

Une première partie décrit succinctement la plateforme de modélisation numérique et sa mise en œuvre. Les résultats principaux de simulation sont ensuite présentés et discutés.

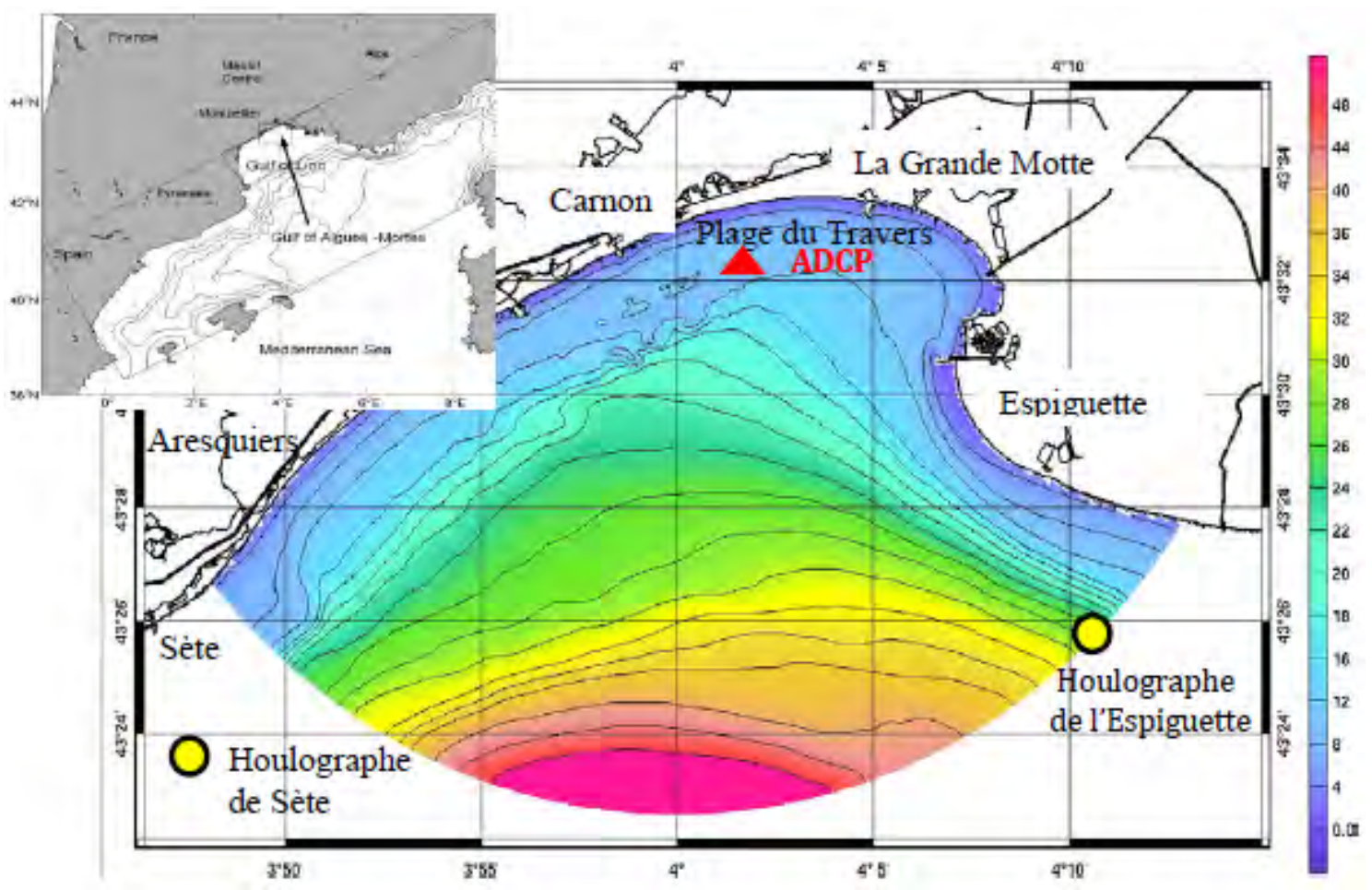

Figure 1. Position et bathymétrie (en $\mathrm{m}$ ) de la zone d'étude. Sites géographiques cités dans le texte. Position des trois stations de mesures.

\section{Description du modèle}

La modélisation numérique multi-échelles vagues/courants/sédiments (MICHAUD et al., 2012 ; ULSES et al., 2008), est basée sur le modèle de circulation océanique côtière Symphonie (MARSALEIX et al., 2008) et le modèle de vagues WaveWatch 3 v4 (ARDHUIN et al., 2010). La validation du modèle Symphonie en zone côtière (e.g. LEREDDE et al., 2007) est étendue à la zone de déferlement des vagues (MICHAUD et al., 2012) suivant les formulations de ARDHUIN et al. (2008) et BENNIS et al. (2011). Le modèle Symphonie est couplé avec un modèle de transport sédimentaire à travers une équation d'advection-diffusion et un module de couche limite de fond qui calcule la 


\section{Thème 2 - Dynamique sédimentaire}

tension turbulente moyenne et maximale sur un cycle de vague au niveau du fond. Le modèle de transport sédimentaire est décrit dans ULSES et al. (2008).

Afin de correctement modéliser la circulation et le transport sédimentaire à la fois en zone côtière sur le plateau mais aussi au niveau de la plage du Travers, un système de trois grilles gigognes a été utilisé. Les caractéristiques de ces grilles sont décrites dans le tableau 1. Les deux premières grilles, MED et GDL, couvrent respectivement la Méditerranée Nord Occidentale et le Golfe du Lion. La dernière grille, GAM, est curvilinéaire avec une résolution allant de $210 \mathrm{~m}$ au large et $8 \mathrm{~m}$ au niveau de la plage.

Tableau 1. Caractéristiques des grilles de calcul.

\begin{tabular}{lllllcl}
\hline Grille & Résolution & Latitude & Longitude & Nlignes & Ncolonnes & $\Delta t(s)$ \\
\hline$M E D$ & $0.1^{\circ}$ & $31^{\circ} \mathrm{N}$ à $45^{\circ} \mathrm{N}$ & $5.6^{\circ} \mathrm{W}$ à $16.3^{\circ} \mathrm{E}$ & 141 & 220 & 400 \\
$G D L$ & $0.02^{\circ}$ & $41.28^{\circ} \mathrm{N}$ à $44.45^{\circ} \mathrm{N}$ & $2.02^{\circ} \mathrm{E}$ à $11.86^{\circ} \mathrm{E}$ & 117 & 213 & 300 \\
$G A M$ & $8 m$ à $210 \mathrm{~m}$ & $43.376^{\circ} \mathrm{N} \grave{a} 43.56^{\circ} \mathrm{N}$ & $3.783^{\circ} \mathrm{E}$ a $4.254^{\circ} \mathrm{E}$ & 57802 noeuds & 5 \\
\hline
\end{tabular}

La bathymétrie (figure 1 pour la grille GAM) est une bathymétrie composite composée de la bathymétrie de l'Ifremer à $200 \mathrm{~m}$ de résolution au large (BERNÉ et al., 2002) et d'une bathymétrie LIDAR réalisée en 2009 par la DREAL. La granulométrie a été spécifiée à partir de prélèvements sédimentaires effectués en 2009 et a permis de compléter la carte de granulométrie réalisée par ULSES (2005) pour le Golfe du Lion. Alors que de nombreuses possibilités de découpages en classes granulométriques sont offertes dans le modèle, nous avons adopté ici 5 classes granulométriques allant des fines aux sables grossiers (silts, argiles, sables fins, sables moyens et sables grossiers) (voir répartition sur la figure 2).

Nous nous sommes intéressés à la période de fin décembre 2008, car nous disposions des mesures hydrodynamiques sur la plage de Sète ayant permis de valider notre modèle sur la zone de déferlement des vagues pendant la même période (MICHAUD et al., 2013). Pendant cette période, une tempête caractérisée par une houle longue de sud-est, de $12 \mathrm{~s}$ de période et $3 \mathrm{~m}$ de hauteur significative s'est produite à partir du 25 décembre. Le vent local était un vent de nord au début de la tempête qui a tourné à partir du 30 décembre pour devenir un vent d'est-sud-est pouvant atteindre dans le GAM, $12 \mathrm{~m} / \mathrm{s}$. Cette tempête est assez caractéristique des tempêtes hivernales dans la région.

\section{Résultats de modélisation}

Nous regardons ici les effets de la tempête de décembre 2008 sur la circulation côtière et littorale ainsi que sur le déplacement des sédiments.

A l'apex de la tempête, des vagues de hauteurs significatives supérieures à $4.5 \mathrm{~m}$ au large entrent dans le GAM (voir figure 3a). Au niveau de la zone dont la bathymétrie est comprise entre 20 et $10 \mathrm{~m}$, des franges de réfractions se forment. Ce phénomène de réfraction est également visible en observant la direction de la vitesse de Stokes des 


\section{XIII ${ }^{\text {èmes }}$ Journées Nationales Génie Côtier - Génie Civil \\ Dunkerque, 2-4 juillet 2014}

vagues (voir figure 3b). Par ailleurs, on observe une décroissance de la hauteur des vagues à mesure qu'elles se rapprochent de la côte, liée à une dissipation par déferlement et friction sur le fond.
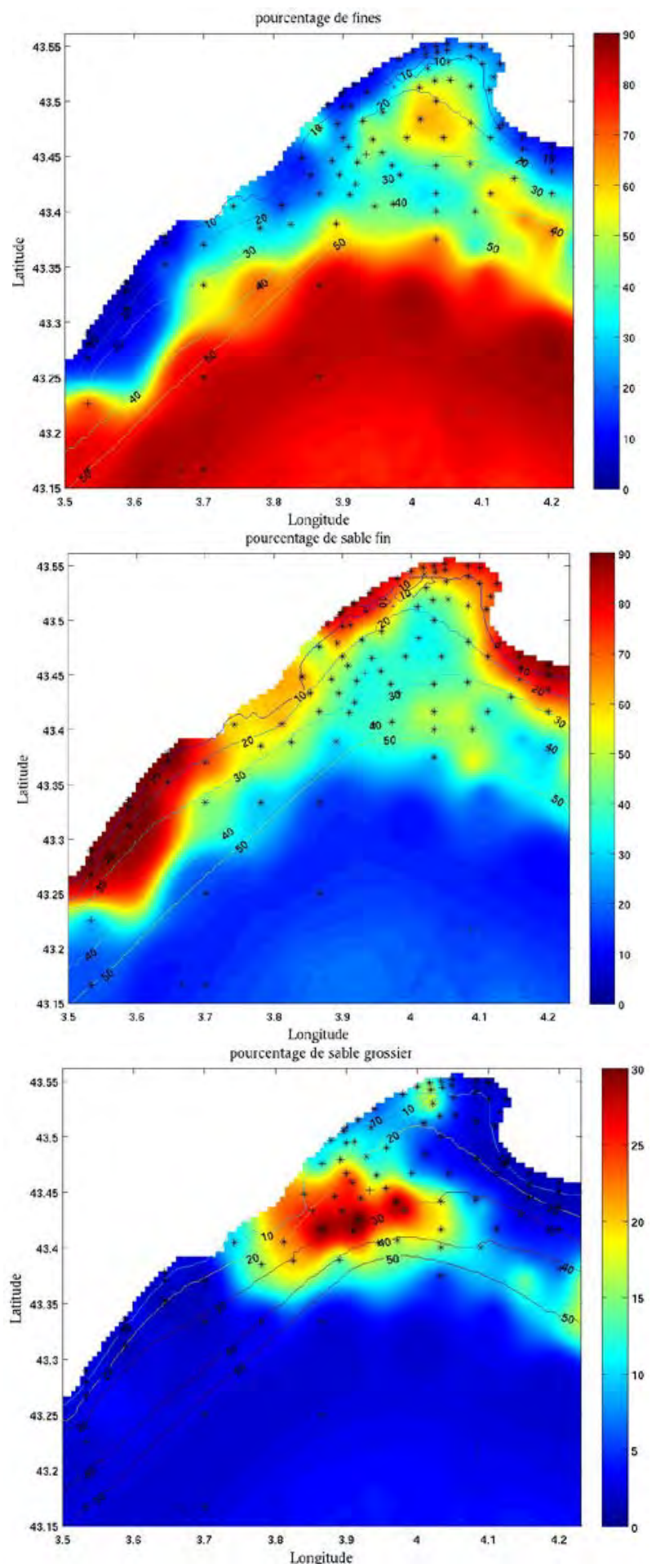

Figure 2. Proportion de fines, de sables fins et de sables grossiers, dans le GAM à partir de prélèvements sédimentaires et d'analyses granulométriques réalisées en 2009. 

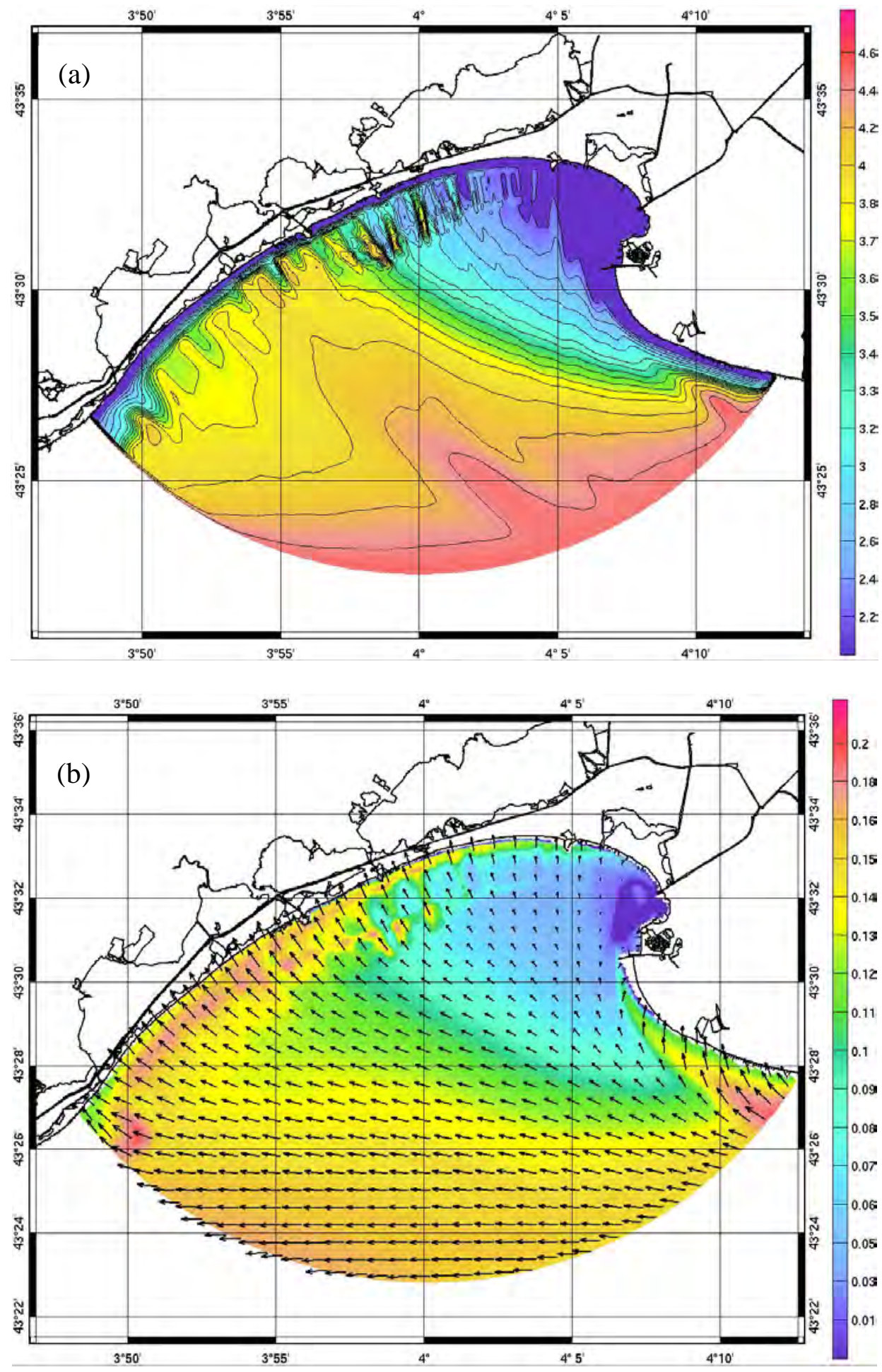

Figure 3. Hauteur significative des vagues (en m) (a) et vitesses de Stokes en surface (en $\mathrm{m} / \mathrm{s}$ ) (b) à l'apex de la tempête (le 26 décembre 2008 à 19h). 


\section{XIII ${ }^{\text {èmes }}$ Journées Nationales Génie Côtier - Génie Civil \\ Dunkerque, 2-4 juillet 2014}

Pendant cet épisode de tempête, les courants induits sont relativement forts (figure 4). Un jet côtier principalement généré par le vent régional se développe dans la zone côtière, longeant l'isobathe $30 \mathrm{~m}$ et se dirigeant vers le sud-ouest (figure $4 \mathrm{a}$ ). Près de la côte, des dérives littorales orientées vers l'est ou l'ouest sont également générées, leurs directions dépendant de l'angle d'incidence des vagues par rapport à la côte. Au niveau de la plage du Travers, la dérive à l'est de Carnon est orientée vers l'est, avec des intensités atteignant $0.50 \mathrm{~m} / \mathrm{s}$ en surface, et la dérive à l'ouest de la Grande Motte, moins intense, est orientée vers l'ouest. Au niveau de la longitude $4^{\circ} 02^{\prime}$, lorsque les deux dérives se rencontrent, un courant orienté vers le large est alors simulé (figure 4b). Près du fond, le courant et les dérives sont moins réguliers (figure 4c), et de multiples courants cross-shore se développent (figure 4d).

Les courants près du fond et les vitesses orbitales de la houle (non montrées) induisent des tensions de cisaillement sur le fond. A partir de ces variables, on peut calculer les tensions de cisaillement moyennes et maximales exercées par les effets conjugués de la houle et des courants. La tension moyenne conditionne le profil du courant près du fond et détermine la diffusion de la matière dans la colonne d'eau tandis que la tension maximale détermine l'érosion du sédiment. Les formulations utilisées sont adaptées de SOULSBY et al. (1995). Les tensions de cisaillement exercées sur le fond (non montrées), peuvent facilement atteindre $15 \mathrm{~N} / \mathrm{m}^{2}$ pendant la tempête dans les zones de bathymétrie inférieure à $10 \mathrm{~m}$. Au niveau du plateau des Aresquiers, de la pointe de l'Espiguette, des franges de réfraction et dans la zone littorale, la tension de cisaillement maximale est particulièrement forte et par conséquent favorise une forte mise en suspension (figure 5). La matière en suspension (MES) peut atteindre jusqu'à $3 \mathrm{~kg} / \mathrm{m}^{2}$ mais est surtout confinée près de la côte, pour des profondeurs inférieures à $20 \mathrm{~m}$. On constate que la zone où la concentration en MES est la plus forte correspond à la zone de déferlement des vagues. On note également que le courant cross-shore observé à la figure 4c est générateur d'une remise en suspension. Ainsi, les sédiments sont transportés dans une direction parallèle à la plage par la dérive littorale, mais également poussés vers le large par des courants d'arrachement. 


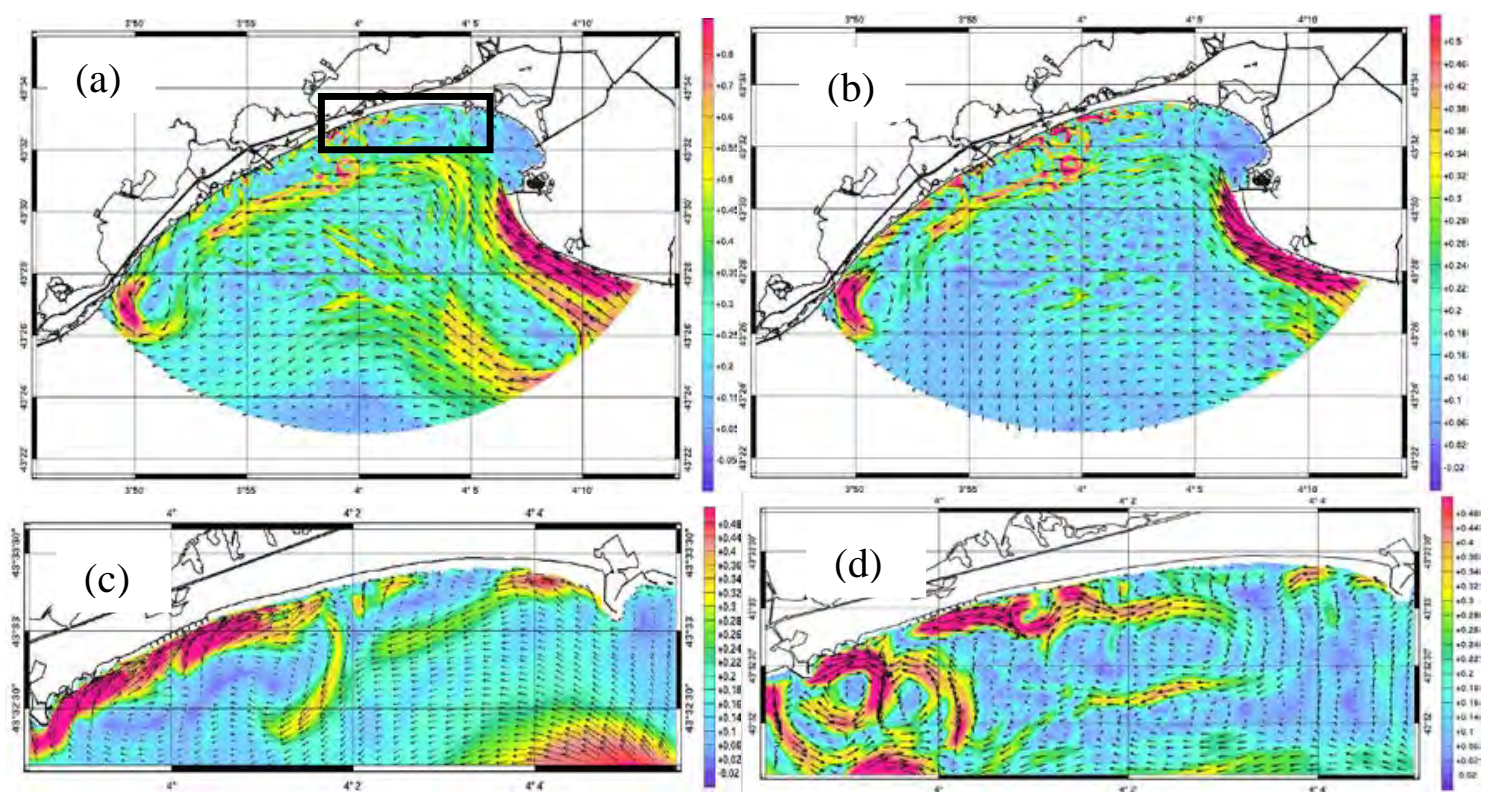

Figure 4. Courants (en m/s) à l'apex de la tempête(le 26 décembre 2008 à 19h), en surface ((a) et (c)) et près du fond ((b) et (d)). Zoom sur la zone de plage du Travers ((c) et (d)).

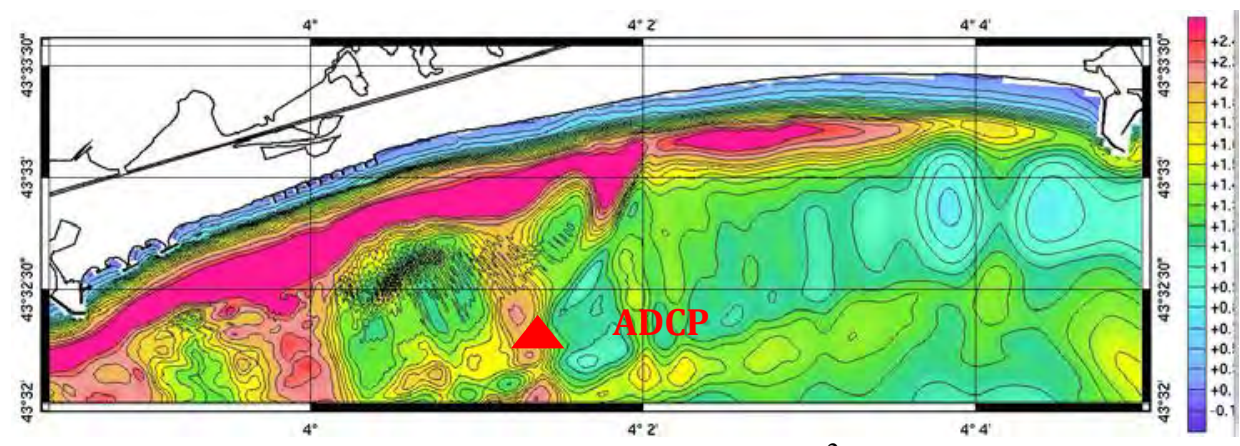

Figure 5. Matière en suspension totale (en $\left.\mathrm{kg} / \mathrm{m}^{2}\right)$ à l'apex de la tempête (le 26 décembre 2008 à 19h).

La représentation de la figure 5 intègre la donnée de la surface jusqu'au fond. On peut toutefois préciser que la MES est en général 100 fois moins forte près de la surface que près du fond. On peut également suivre l'évolution de toutes ces variables et en particulier de la MES en fonction du temps pendant la tempête (résultats non montrés). Pendant le tombant de la première période de la tempête, après le premier pic (le soir du 26/12), la concentration en MES diminue rapidement, pour être très faible au bout de deux jours $\left(<1 \mathrm{~kg} / \mathrm{m}^{3}\right)$ dans la zone très littorale ( $<3 \mathrm{~m}$ de fond). La figure 6 montre le profil vertical suivant le temps de la MES sous $10 \mathrm{~m}$ de fond, au point noté "ADCP" sur les figures 1 et 5 . On note que la matière est mise en suspension pendant la tempête (lorsque les vagues ont une $H s>2 \mathrm{~m}$ ) puis la concentration subit une décroissance rapide dans les deux jours suivants. Cette tempête a donc accru la turbidité de la région littorale 


\section{XIII ${ }^{\text {èmes }}$ Journées Nationales Génie Côtier - Génie Civil \\ Dunkerque, 2-4 juillet 2014}

surtout à l'apex de la tempête. Les herbiers de Posidonies, étant situés sous $10 \mathrm{~m}$ de fond, au niveau de l'ADCP, sont alors impactés par cette remise en suspension. Les durées de périodes turbides sont toutefois très courtes (quelques heures) et il conviendra d'évaluer ces durées pour de plus fortes tempêtes. La question de l'impact sur la capacité photosynthétique et la santé des herbiers reste toutefois bien posée.

Outre le calcul de la MES, le modèle de transport sédimentaire permet de calculer les modifications géomorphologiques. La figure 7 représente le bilan sédimentaire entre le 21/12/2008 et le 01/01/2009, toutes classes de sédiments confondus (des silts aux sables grossiers, 5 classes sont représentées dans le modèle). On constate que la tempête a généré de nombreux changements, principalement en deçà de $15 \mathrm{~m}$ de bathymétrie. En zone littorale au niveau de la plage du Travers, on observe un phénomène de recul des barres internes, surtout au niveau des épis de Carnon (voir figure 7). En effet, sous de très petit fonds, une érosion a lieu, puis sous $\sim 3 \mathrm{~m}$ de fond (au niveau de la barre interne), on observe une accrétion, puis en allant vers le large à nouveau une zone en érosion. Cela traduit en réalité une migration des barres sédimentaires vers le large connu et observé lors des épisodes de tempête. Un travail de validation reste toutefois à réaliser et nous n'irons pas plus loin ici dans l'interprétation. Ainsi, la dérive littorale est à la fois génératrice de transport longshore redistribuant le sédiment parallèlement à la côte, et au gré des digues et épis, mais aussi d'un transport cross-shore (courant de retour près du fond) capable de déplacer les barres sédimentaires. Les multiples recirculations (figure 4d) sont, elles aussi, à même de transporter vers le large les sédiments.

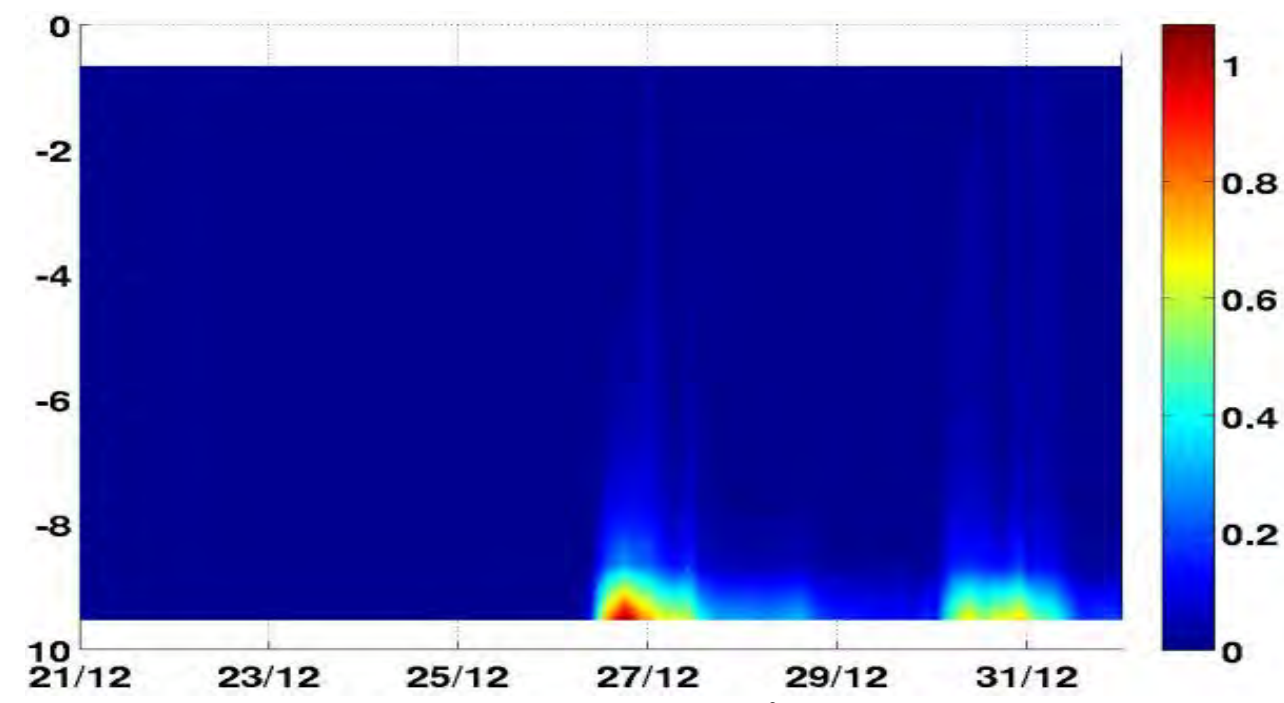

Figure 6. Matière en suspension totale (en $\mathrm{kg} / \mathrm{m}^{3}$ ) sous $10 \mathrm{~m}$ de fond à la position de l'ADCP, en fonction de la profondeur (m) et du temps (du 21/12/2008 au 01/01/2009). 


\section{Perspectives et conclusion}

La chaine de calculs des flux atmosphériques, vagues, courants, transports sédimentaires, évolution morphologique est aujourd'hui bien en place et validée (MICHAUD et al., 2012 \& 2013). Elle peut permettre de simuler des épisodes de tempête tels que celui de fin décembre 2008 présenté dans cet article. Le modèle donne également d'autres éléments non montrés ici comme le devenir des sédiments répartis en différentes classes de taille. En effet, les panaches sédimentaires sont avant tout constitués de particules fines y compris de sables fins qui constituent une partie du stock rechargé sur les plages. L'étape de validation spécifique à ce site de la plage du Travers est en cours. En effet, des campagnes de mesures hydrosédimentaires et bathymétriques ont pu être réalisées de janvier à mai 2012 avec un épisode significatif de tempête fin avril 2012. Un ADCP (placé au point noté ADCP sur les figures 1 et 5) a ainsi pu mesurer les courants et les caractéristiques de vagues sur cette période. Nous bénéficions également des enregistrements des houlographes de Sète et de l'Espiguette (voir figure 1) dans cette zone. Ces données seront utiles à la validation du modèle hydrodynamique. Des données de turbidité et de de bathymétrie permettront d'évaluer la partie portant sur les transports sédimentaires et la morphodynamique. Une fois bien validé, ce modèle pourra être utilisé en mode prospectif pour simuler l'impact de tempêtes plus énergétiques susceptibles de produire plus de MES et plus de remaniement des fonds.

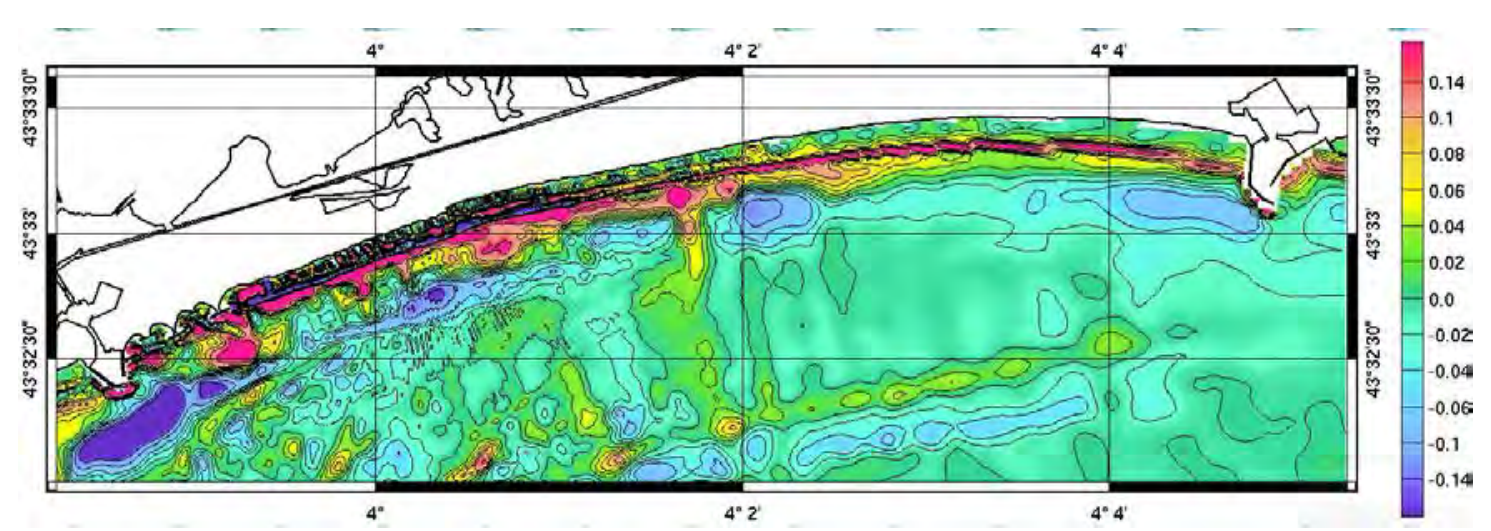

Figure 7. Bilan érosion (valeurs négatives) vs. dépôt (valeurs positives) (en m) entre le 21/12/2008 et le 01/01/2009.

\section{Remerciements}

Le projet REPBAM a été financé sur fonds européens et régionaux FEDER, sur fonds de l'état français (FNADT) et sur ressources propres de l'Université Montpellier 2. Nous remercions la DREAL Languedoc-Roussillon pour la bathymétrie LIDAR et Météo-France pour les données du modèle Aladin. Le modèle de circulation océanique côtière Symphonie est développé par la plateforme Sirocco. 


\section{XIII ${ }^{\text {èmes }}$ Journées Nationales Génie Côtier - Génie Civil \\ Dunkerque, 2-4 juillet 2014}

Les sources du code sont disponibles sur

http://sirocco.omp.obs-mip.fr/outils/Symphonie/Sources/SymphonieSource.htm

\section{Références bibliographiques}

ARDHUIN F., RASCLE N., BELIBASSAKIS K.A. (2008). Explicit wave-averaged primitive equations using a generalized Lagrangian mean. Ocean Modelling, Vol. 20(1), pp 35-60. http://dx.doi.org/10.1016/j.ocemod.2007.07.001

ARDHUIN F., ROGERS E., BABANIN A., FILIPOT J.F., MAGNE R., ROLAND A., WESTHUYSEN A., QUEFFEULOU P., LEFEVRE J.M., AOUF L., COLLARD F. (2010). Semiempirical Dissipation Source Functions for Ocean Waves. Part I: Definition, Calibration, and Validation. Journal of Physical Oceanography, Vol. 40(9), pp 1917-1941. http://dx.doi.org/10.1175/2010JPO4324.1

BERNÉ S., CARRÉ D., LOUBRIEU B., MAZÉ J.P., MORVAN L., NORMAND A. (2002). Carte morpho-bathymétrique du golfe du Lion au 1/250 000e. Editions de l'Ifremer.

BENNIS A.C., ARDHUIN F., DUMAS F. (2011). On the coupling of wave and threedimensional circulation models: Choice of theoretical framework, practical implementation and adiabatic tests. Ocean Modelling, Vol. 40(3-4), pp 260-272. http://dx.doi.org/10.1016/j.ocemod.2011.09.003

LEREDDE Y., DENAMIEL C., BRAMBILLA E., LAUER-LEREDDE C., BOUCHETTE F., MARSALEIX P. (2007). Hydrodynamics in the Gulf of AiguesMortes, NW Mediterranean Sea: in situ and modelling data. Continental Shelf Research, Vol. 27, pp 2389-2406. http://dx.doi.org/10.1016/j.csr.2007.06.006

MARSALEIX P., AUCLAIR F., FLOOR J.W., HERRMANN M.J., ESTOURNEL C., PAIRAUD I., ULSES C. (2008). Energy conservation issues in sigma-coordinate freesurface ocean models. Ocean Modelling, Vol. 20, pp 61-89. http://dx.doi.org/10.1016/j.ocemod.2007.07.005

MICHAUD H., MARSALEIX P., LEREDDE Y., ESTOURNEL C., BOURRIN F., LYARD F., MAYET C., ARDHUIN F. (2012). Three-dimensional modelling of waveinduced current from the surf zone to the inner shelf. Ocean Science, Vol. 8, pp 657-681. http://dx.doi.org/10.5194/os-8-657-2012

MICHAUD H., ROBIN N., ESTOURNEL C., MARSALEIX P., LEREDDE Y., CERTAIN R., BOUCHETTE F. (2013). 3D Hydrodynamic modelling of a microtidal barred beach (Sète, NW Mediterranean Sea) during storm conditions. Actes du colloque Coastal Dynamics 2013. 24-28 juin 2013, Arcachon, France.

SHORT F.T., WYLLIE-ECHEVERRIA S. (1996). A Review of Natural and Humaninduced Disturbance of Seagrasses. Environmental Conservation, Vol. 23(1), pp 17-27. http://dx.doi.org/10.1017/S0376892900038212 
Thème 2 - Dynamique sédimentaire

SOULSBY R., STIVE M., DE VRIEND H., FREDSOE J., HAMM L., TEISSON C., WINTERWERP J. (1995). Bed shear stresses due to combined waves and current. Advances in coastal morphodynamics, pp 4-20 4-23.

ULSES C. (2005). Dynamique océanique et transport de la matière particulaire dans le Golfe du Lion : crue, tempête et période hivernale. Thèse. Université Paul Sabatier, Toulouse.

ULSES C, ESTOURNEL C., DURRIEU DE MADRON X., PALANQUES A. (2008). Suspended sediment transport in the gulf of Lions (NW Mediterranean): Impact of extreme storms and floods. Continental Shelf Research, Vol. 28, pp 2048-2070. http://dx.doi.org/10.1016/j.csr.2008.01.015 